Methods The retrospective review of the institutional, IRBapproved database was undertaken to find cases where the TracStar LDP $^{\mathrm{TM}}$ or Zoom ${ }^{\mathrm{TM}} 88$ guide catheters (Imperative Care, Campbell, CA) were used with a TRA for neurointerventions. For this study, gender, age, case type, target anatomy, distal location reached with the guide catheters, time from access to reperfusion, complications, and the Thrombolysis in Cerebral Infarction (TICI) score were collected. Safe placement of the guide catheters to the target anatomy was considered a technical success.

Results From August 2020 to March 2021, 13 patients underwent TRA neurointerventions using the TracStar LDP or Zoom 88 guide catheters. The TracStar LDP was used in 77\% $(10 / 13)$ of patients; the Zoom 88 guide catheter was used in $23 \%(3 / 13)$ of patients. The type of intervention was acute ischemic stroke in $69 \%(9 / 13)$ of patients and aneurysm embolization in $31 \%(4 / 13)$ of patients. The TracStar LDP facilitated the implantation of flow diverters in all aneurysm cases with 75\% (3/4) using a biaxial system. Overall, there was an even distribution between females $(54 \%, 7 / 13)$ and males $(46 \%, 6 / 13)$. The median age was 72.5 (range $=48-88$ ) years. Most patients with aneurysms were females (75\%, 3/4); this population's median age was 58.5 (range $=54-64$ ) years. In patients with acute ischemic stroke, the median age was 76 (range $=48-88$ ) years. The target anatomy for all aneurysm patients was the left internal carotid artery. The target anatomy for stroke patients included right internal carotid artery in $77.8 \%(7 / 9)$ of patients, left internal carotid artery in $11.1 \%(1 / 9)$ of patients and left vertebral artery in $11.1 \%$ (1/ 9) of patients. In these patients, the final positions of the guide catheters included Supraclinoid Carotid $(n=2)$, Ophthalmic segment $(n=2)$, Petrous Carotid, Petro-Cavernous junction, Basilar, Cavernous-Carotid, and in one case the Zoom 88 was positioned in the right $\mathrm{M} 1$. The median puncture to reperfusion time was 30.5 (range $=5-50$ ) minutes. The TICI $2 \mathrm{~b}$ or greater was achieved in $77.8 \%$ (7/9) of patients. There were no complications associated with the guide catheters. In two patients (one stroke, one aneurysm), the TracStar LDP guide catheter was used successfully as the rescue option after the initial approach with the 0.079 " radial guide catheter failed.

Conclusion Transradial access with the TracStar LDP and Zoom 88 large-bore guide catheters is feasible and safe in achieving intracranial access for neurointerventions in carefully selected patients.

Disclosures L. Lyons: None. M. Abouelleil: None. A. Restrepo Orozco: None. L. Verhey: None. J. Tsai: 2; C; Cerenovus, Medtronic. P. Mazaris: 2; C; Stryker. J. Singer: 2; C; Medtronic, Cerenovus, Stryker, Pneumbra, Nico.

\section{E-083 SAFETY AND EFFICACY OF XACT STENT IN TANDEM INTERNAL CAROTID ARTERY AND INTRACRANIAL LARGE VESSEL OCCLUSION}

M Oliver*, G Dawod, S Zaidi, M Jumaa. University of Toledo/Promedica, Toledo, $\mathrm{OH}$

10.1136/neurintsurg-2021-SNIS. 178

Introduction Endovascular stenting of the extracranial internal carotid artery (ICA) in the setting of tandem intracranial arterial occlusion (ICAO) is an area of ongoing research.

Objective We demonstrate the safety and efficacy of endovascular stenting of the ICA with tandem ICAO using the Xact stent.
Design This is a retrospective cohort analysis of all patients presenting to a single academic center with tandem ICA/ICAO who underwent mechanical thrombectomy from 2015-2020. Patients were included per AHA guidelines excluded if preprocedural angiography did not show tandem occlusions.

Main outcomes and measures Baseline variables included age, baseline National Institute of Health Stroke Scale (NIHSS), baseline Alberta Stroke Program Early CT Score (ASPECTS), site of intracranial occlusion, treatment techniques and time efficiencies, and thrombolysis in cerebral infarction score. Outcome measures included modified Rankin scale (mRs) at 90 days, median infarct volume, stent complications, stent restenosis rate, recurrent stroke at 30 days and symptomatic intracerebral hemorrhage (sICH).

Results A total of 67 patients with symptomatic angiographically-confirmed tandem ICA/ICAO were identified. The median patient age was 66, baseline median NIHSS was 16.2, Mean ASPECTS 8.1, ICAO location was the M1 segment of middle cerebral artery (MCA) in 39\% of patients, internal carotid artery terminus in 37\%, M2 segment of MCA in $23 \%$ and M3 segment of MCA in $1.6 \%$ of patients. Successful reperfusion of TICI 2B-3 was achieved in 91\% of patients. Favorable mRS of $0-2$ was achieved in 52\% of cases, mortality was $19 \%$, and median final infarct volume was $30.9 \mathrm{~mL}$. The rate of stent complications occurred in 3\% of patients, most common being partial in stent thrombosis extending intraluminally. Rate of sICH was 4\%, recurrent stroke occurred in $5 \%$ of patients within 30 days, and in-stent stenosis $>50 \%$ happened in $16 \%$ at 18 months. No patients required additional interventions.

Conclusions The Xact carotid stent is safe and efficacious in the treatment of tandem ICA/ICAO lesions. Larger prospective trials are needed to help confirm our retrospective findings.

Disclosures M. Oliver: None. G. Dawod: None. S. Zaidi: None. M. Jumaa: None.

\section{E-084 NICARDIPINE VERSUS CLEVIDIPINE FOR POST MECHANICAL THROMBECTOMY BLOOD PRESSURE MANAGEMENT IN PATIENTS WITH ISCHEMIC STROKE DUE TO ISOLATED MIDDLE CEREBRAL ARTERY OCCLUSION}

${ }^{1} \mathrm{M}$ Oliver*, ${ }^{1} \mathrm{~J}$ Shawwer, ${ }^{1} \mathrm{H}$ Salahuddin, ${ }^{2} \mathrm{~S}$ Desai, ${ }^{1} \mathrm{~S}$ Zaidi, ${ }^{1} \mathrm{M}$ Jumaa. ${ }^{1}$ University of Toledo/ Promedica, Toledo, $\mathrm{OH}^{2}{ }^{2}$ Barrow Neurological Institute, Phoenix, AZ

\subsection{6/neurintsurg-2021-SNIS. 179}

Objective Intensive blood pressure (BP) management after mechanical thrombectomy (MT) may be beneficial in patients with acute anterior circulation (AC) Ischemic stroke (IS) due to large vessel occlusion (LVO). We sought to evaluate the efficacy of Nicardepine (NCR) vs. Clevidipine (CLV) in IS patients with LVO who underwent successful MT.

Methods With IRB approval, we retrospectively collected data on consecutive patients with isolated MCA M1 occlusion who underwent successful MT (TICI score of 2B or 3). Patient demographics, baseline characteristics, time efficiencies, procedural data, incidence of hemorrhagic transformation (HI) and symptomatic ICH per ECASS II criteria on $24 \mathrm{H}$ head CT, and clinical outcomes were recorded. We also recorded duration from recanalization to optimal BP control (ROBP), IV drip utilized (NCR vs. CLV), number of SBP excursions, and total duration of time outside the target SBP in first $24 \mathrm{H}$. 
Results We identified 111 patients between 2017 and 2020, 57 patients required a drip (43 NCR, $14 \mathrm{CLV})$, and 54 patients were managed with prn medications. Median NIHSS was higher in the drip group (18, IQR 13-21) vs. 15.5 (IQR $9-20), p$ value $=0.03$. There was no significant difference in the rate of good clinical outcome defined as mRs of $0-2$ or return to baseline $(47.4$ vs $61.1 \%, \mathrm{p}$ value $=0.18)$; rate of any HI $(29.8 \%$ vs. $27.8 \%$, p value $=0.84)$ and $\operatorname{siCH}(5.3 \%$ vs $0 \%$, $\mathrm{p}$ value $=0.24$ ) between the two groups (drip vs. no drip). When comparing the NCR to CLV groups, median ROBP was significantly shorter in the CLV group, $5 \mathrm{~min}$ (IQR 0.75-7) vs. $17 \mathrm{~min}$ (IQR 6-35), $\mathrm{p}$ value $=0.003$; and total duration of time outside the pre-specified BP range was $37.5 \mathrm{~min}$ (IQR $19-120)$ vs. $118 \mathrm{~min}(57-227)$ consecutively, $\mathrm{p}$ value $=0.045$. HT rates $(14.3 \%$ vs. $34.9 \%$, p value $=0.19)$, sICH rates $(0$ vs. $7 \%, \mathrm{p}$ value $=0.57)$, and rate of good clinical outcome $(64.3 \%$ vs. $41.9 \%, \mathrm{p}$ value $=0.22$ ) were similar between the two groups (CLV vs. NCR). In a Multivariate analysis, after adjusting for age, gender, IV tPA administration, drip used and time out of range; NIHSS (OR 0.8, CI 0.70-0.91, p=0.0008) and ROBP $(\mathrm{OR}=1.05$, CI 1.0-1.1, p value $=0.032)$ were independent predictors of good clinical outcome.

Conclusion CLV achieved faster ROBP time and better BP control after MT. Further prospective studies are needed.

Disclosures M. Oliver: None. J. Shawver: None. H. Salahuddin: None. S. Desai: None. S. Zaidi: None. M. Jumaa: None.

\section{E-085 INTERVENTIONAL AND DIAGNOSTIC NEURORADIOLOGY FELLOWSHIP EDUCATION IN THE COVID-19 ERA}

'Z Wilseck*, ${ }^{2} S$ Bamezai, ${ }^{2} \mathrm{~N}$ Novakovic, ${ }^{3} \mathrm{~A}$ Copelan, ${ }^{4} \mathrm{~J}$ Wilseck, ${ }^{1} \mathrm{~A}$ Srinivasan, ${ }^{5} \mathrm{~A}$ Pandey, ${ }^{5} \mathrm{G}$ Thompson, 'J Gemmete, ${ }^{1} \mathrm{~N}$ Chaudhary. ${ }^{1}$ Radiology, University of Michigan Health System, Ann Arbor, Mli ${ }^{2}$ University of Michigan Health System, Ann Arbor, MI; ${ }^{3}$ Radiology, Consulting Radiologists Ltd, Edina, MN; ${ }^{4}$ Radiology, Oakland University William Beaumont Hospital, Royal Oak, MI; Neurosurgery, University of Michigan Health System, Ann Arbor, MI

\subsection{6/neurintsurg-2021-SNIS. 180}

Background and Purpose Given the relatively short duration and multiple facets of education, both interventional and diagnostic neuroradiology fellowships must be used efficiently. As hospital systems utilize resources to provide clinical care of patients with COVID-19, the overall number of elective neurointerventional procedures has been limited and the overall volume of diagnostic imaging has decreased. This article will evaluate and discuss the impact of procedural volumes between two academic centers, the impact on overall diagnostic neuroradiology imaging volume, and the rapid migration to online web-based education and research collaboration on both interventional and diagnostic neuroradiology fellowship experiences in the COVID-19 era.

Material and methods A retrospective review was conducted at two academic tertiary care hospitals to evaluate the change in overall elective and emergent neurointerventional case volume during the COVID-19 pandemic spanning from January 2020 - April 2020 and those volumes were compared to preCOVID-19 case volume during January 2019-April 2019. A single center comparison of annual neurointerventional case numbers during 2019 and 2020 was performed with sub-analyses of case types. Annual diagnostic neuroradiology imaging volume was also evaluated for 2019 and 2020.

Results Both tertiary care academic medical centers experienced a statistically significant decrease in the number of elective neurointerventional cases between March - April 2019 and March - April 2020. There was also a statistical different decrease in elective case volume during the fourth quarter of the 2020 academic year (April - June). A statistically significant decrease in case volume was not seen in the setting of thrombectomy for acute ischemic stroke. A statistically significant decrease in aneurysmal subarachnoid hemorrhage (aSAH) case volume was only seen at site \#2. Between 2019 and 2020 there was a $32 \%$ drop in neuroradiology case volume during the fourth quarter of the 2020 academic year (April June). Similar sizable decreases in diagnostic neuroradiology case volume were not appreciated in the remaining quarters of 2019 and 2020.

Conclusion Elective neurointerventional case numbers decreased as a result of the COVID-19 pandemic, particularly impacting the fourth quarter of the 2019-2020 academic year (April - June). Similarly, there was a sizable decrease in diagnostic neuroradiology case volume during this same time period. The decrease in procedural and diagnostic neuroradiology cases raised concerns regarding the educational experience for both diagnostic and neurointerventional radiology fellows. However, as a result of decreased case volume, fellowship education was rapidly augmented with a wide variety of online webinars, lectures, case conferences, and research collaborations.

Disclosures Z. Wilseck: None. S. Bamezai: None. N. Novakovic: None. A. Copelan: None. J. Wilseck: None. A. Srinivasan: None. A. Pandey: None. G. Thompson: None. J. Gemmete: None. N. Chaudhary: None.

\section{E-086 HUMAN INTRACEREBRAL HEMORRHAGE (ICH): EARLY HEMOLYSIS/ERYTHRYOLYSIS, IRON OVERLOAD, PERIHEMATOMAL EDEMA \& SURVIVING WHITE MATTER; TRANSLATIONAL EVIDENCE FOR BRAIN TISSUE INJURY MARKERS ON MRI}

${ }^{1} \mathrm{~N}$ Novakovic, ${ }^{2} \mathrm{Z}$ Wilseck*, ${ }^{2} \mathrm{~T}$ Chenevert, ${ }^{3} \mathrm{G} X \mathrm{X},{ }^{3} \mathrm{R}$ Keep, ${ }^{3} \mathrm{~A}$ Pandey, ${ }^{2} \mathrm{~N}$ Chaudhary. ${ }^{1}$ University of Michigan Health System, Ann Abor, MI; ${ }^{2}$ Radiology, University of Michigan Health System, Ann Abor, Ml; ${ }^{3}$ Neurosurgery, University of Michigan Health System, Ann Abor, MI

\subsection{6/neurintsurg-2021-SNIS. 181}

Objective Intracerebral Hemorrhage (ICH) in humans has not benefited from any translation from the benchside to the bedside in terms of established therapy or prognostic markers to guide treatment. ICH continues to inflict devastating neurological consequences. There is gatheringevidence for some MRI parameters to reflect the amount of iron that leaches out of the hematoma to cause neurotoxicity. We examine the role of early erythrolysis within the hematoma as determined by MRI in ICH patients and its role in predicting iron overload to the surrounding tissues and its effects on the surrounding white matter based on MRI signal quantification.

Methods All patients recruited in the study since 2017 were included in the analysis. A total of 15 patients were prospectively enrolled into the NIH funded study since 2017 with informed consent and local IRB approval. The patients had MRIs at day $1 \& 3$, termed as early, day 14 designated as subacute period and day 30 as the late period. All MRIs were performed on a 3 Tesla MRI. Analysis and measurements were performed on $\mathrm{T} 2 *$ maps, relaxivity (R2*) maps, fractional anisotropy (FA) maps, and edema extent on T2 or FLAIR sequences. Individual measured parameter was then 